\title{
Controversies about the central compartment in thyroid cancer
}

\author{
Editorial regarding the article "Clinical impact of cervical lymph node involvement and \\ central neck dissection in patients with papillary thyroid carcinoma: a retrospective \\ analysis of 368 cases" by Alexandre Bozec et al.
}

\author{
Ashok R. Shaha \\ Received: 19 April 2011/Accepted: 6 May 2011/Published online: 1 June 2011 \\ (C) Springer-Verlag 2011
}

\begin{abstract}
Alexandre Bozec et al. [1]. discuss their experience with 368 patients undergoing central compartment neck dissection in this contemporary article. The issue of central compartment neck dissection continues to be controversial in the modern era. The management of thyroid cancer has always been a controversial subject;however, the major thrust of debate has switched from total versus less than total thyroidectomy to the indications for routine prophylactic central compartment neck dissection[2] and the use of postoperative radioactive iodine ablation. These issues have seen a major paradigm shift over the past 10-15 years, primarily due to our ability to detect the early recurrent disease with thyroglobulin assay and routine follow-up ultrasound of the neck.
\end{abstract}

The subject of management of the neck has been extensively debated since the prognostic implications of the presence of positive nodes, either in the central compartment or in the lateral neck, was found to be minimal. Patients do remarkably well with positive neck nodes. In patients below the age of 45, the stage of the tumor does not change from stage I whether the nodes are positive or not. In view of this, prophylactic neck dissection raises a major issue about the long-term effect on prognosis, recurrence, and complications from the surgical procedure itself. It is very important to balance the risk of complications against the long term benefit of any surgical procedure. The authors have reviewed their large experience in a retrospective fashion. This article raises some difficulties in the interpretation and conclusion, primarily since the data are retrospective and the decision regarding

\footnotetext{
A. R. Shaha $(\bowtie)$

Head and Neck Service, Memorial Sloan-Kettering Cancer

Center, 1275 York Avenue, New York, NY 10065, USA

e-mail: shahaa@mskcc.org
}

prophylactic central compartment dissection was the surgeon's choice. The extent of central compartment dissection also remains unclear, and it would be appropriate to assume that, again, it is of the operating surgeon's choice and experience. However, the authors conclude three important features of positive central compartment nodes: the age of the patient, size of the tumor, and the extrathyroidal extension. These are important prognostic features for the possibility of future recurrence with or without central compartment dissection. The major issues concerning any operating surgeon are the risks of elective central compartment dissection, mainly related to transient and permanent recurrent laryngeal nerve injury, and the transient or permanent hypoparathyroidism. In the majority of the series, the incidence of permanent hypoparathyroidism is much higher in this group of patients, even with the dedicated parathyroid autotransplantation.

The authors in this article have frankly reported a higher incidence of temporary and permanent hypoparathyroidism with prophylactic central compartment dissection. Prior to the era of thyroglobulin and ultrasound studies, there was hardly any discussion about the central compartment dissection. This subject appears to be a contemporary physician- and technology-driven problem. In the past, patients were evaluated clinically in follow-up, and unless recurrent disease was clinically palpable, no treatment intervention was undertaken. Patients continued to do well and required surgical intervention only on a therapeutic basis. In 2006, ATA guidelines recommended that the elective central compartment dissection may be considered in patients with papillary carcinoma of the thyroid. This recommendation was used by the proponents of elective central compartment dissection to support their viewpoint. However, it was apparent to the ATA committee that there was no fundamental strong basis for this recommendation, and the 
complication rate was probably unacceptable for this recommendation. In 2009, the ATA guidelines [3] were switched to elective nodal dissection which may be considered in patients with high-risk thyroid cancer, such as large-sized tumor, extrathyroidal extension, or aggressive histology. These appeared to be the sentiments expressed by many surgeons who were against routine prophylactic central compartment dissection. The authors in this manuscript have essentially substantiated the recommendations made by the ATA in 2009.

Certain issues remain unclear in this article. Six patients with permanent recurrent laryngeal nerve palsy, $6 \%$ of patients receiving external radiation therapy, and lack of subanalysis of papillary thyroid carcinoma such as tall cell, insular, or poorly differentiated, fall into a different category of aggressive thyroid cancer requiring aggressive surgical and postoperative treatment with radioactive iodine ablation. Patients requiring external radiation therapy are mostly high risk thyroid cancer patients, and require all the help in the world-surgically and with adjuvant treatment. Their long-term outcome is generally guarded. The authors' conclusions about the high incidence of recurrent disease in patients with the multiple positive nodes is quite intriguing, as a patient with multiple positive nodes is more likely to have an additional positive lymph node left behind during the initial surgical procedure. Our technology has advanced so much that now we are able to identify this minimal volume disease during routine postoperative follow-up. The major issue remaining unclear is the implication of this mini-volume recurrent disease (Table 1) Will it ever become clinically apparent and prognostically significant, or is it a natural history of patients with papillary carcinoma of the thyroid?

The incidence of nodal metastasis in papillary carcinoma of the thyroid extends from $40-70 \%$, even if the disease is clinically non-palpable. In the absence of elective lateral neck dissection, several patients will harbor microscopic metastatic disease to the lymph nodes and live in symbiosis with this microscopic disease. In a very small percentage of patients this disease becomes clinically apparent, requiring surgical intervention [4]. In the 1950s and 1960s, bilateral radical neck dissection was a relatively common surgical procedure. However, over a period of time, surgeons-especially those from Memorial SloanKettering Cancer Center-recognized that this surgical procedure gave no more advantage and only therapeutic neck dissection was advocated. In the last two decades there has been more emphasis on considering central compartment neck dissection and patient follow-up on clinical grounds. In the last 10 years there has been a major emphasis on thyroglobulin and routine ultrasound, identifying recurrent disease in almost one-fourth of these patients (Fig. 1) Whether this number will change remarkably with prophylactic neck dissection remains to be seen. It is almost impossible to remove the last positive node in every patient with positive central or lateral compartment disease. Elective central compartment dissection has higher morbidity mainly related to temporary and permanent hypoparathyroidism. When a patient develops permanent hypoparathyroidism, it may appear that the treatment has been worse than the disease. This reminds us of 'let the punishment fit the crime'. The issue with elective central compartment dissection revolves around the difficulty in preoperative evaluation with the ultrasound of the central compartment in the presence of thyroid gland. Hashimoto's thyroiditis is a fairly common entity in the United States, leading to a high incidence of enlarged nodes in the central compartment. Our general philosophy is to evaluate the central compartment in every patient with papillary carcinoma of the thyroid and to reserve nodal dissection only for patients with high risk primary tumor or suspicious lymph nodes at the time of surgery. We are generally in favor of obtaining a frozen section of a suspicious lymph node, and if positive, then considering appropriate central compartment dissection. Positive Delphian nodes are seen in approximately $25 \%$ of patients with papillary carcinoma of the thyroid, and should be resected if enlarged or suspicious.

The issue of unilateral versus bilateral central compartment dissection continues to be another major subject of controversy. Our general philosophy is to evaluate the both sides and remove only those suspicious lymph nodes. The extent of neck dissection also continues to be a controversial topic. Even though the central compartment is described as the region extending from the hyoid to the innominate vessels, generally there are hardly any enlarged
Table 1 Extent of metastatic disease in neck nodes from papillary carcinoma of the thyroid

\begin{tabular}{ll}
\hline Type & Impact on outcome \\
\hline Micrometastasis & None \\
Mini-metastasis (detected by U/S of Tg) & None \\
Mini-volume metastasis & None \\
Large volume metastasis & Maybe (regional or distant) \\
Major metastasis (aggressive histology) & Yes, older patient (regional or distant) \\
\hline
\end{tabular}




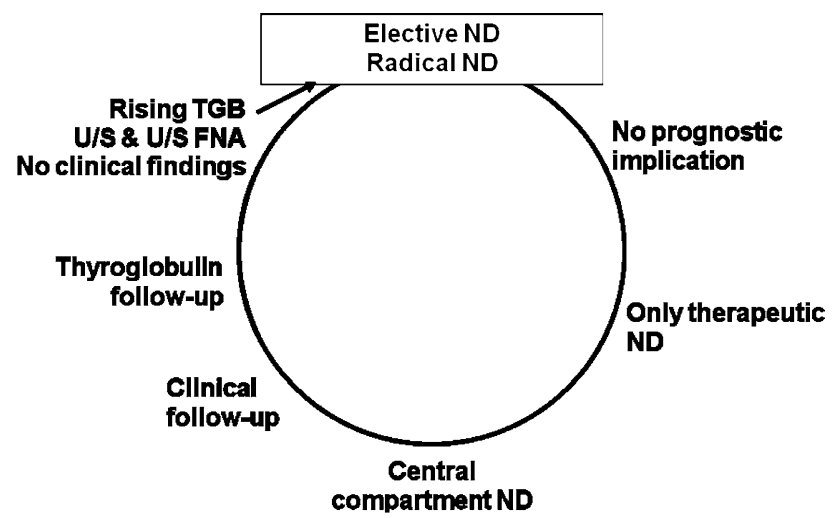

Fig. 1 Full circle in the management of neck nodes in papillary carcinoma of the thyroid

lymph nodes above the upper pole of the thyroid gland and there is hardly any reason to dissect up to the hyoid bone. The lower most extent of dissection remains ambiguous, as there is no defined plane between Level VI and Level VII nodes. However, if there are any suspicious lymph nodes in this area, appropriate paratracheal clearance should be performed as low as possible through the cervical route. Even some of the ardent proponents of the central compartment dissection recently reported no difference in the postoperative thyroglobulin level in patients who had undergone total thyroidectomy alone or total thyroidectomy with central compartment neck dissection [5]. Our ability to detect and localize the minute recurrent disease may be harmful to some patients undergoing surgery for recurrent small volume disease. Our general practice is not to perform a fine needle aspiration biopsy unless the suspicious lymph nodes are larger than $1 \mathrm{~cm}$. It is quite likely that this mini-volume disease may be difficult to localize at the time of reoperative surgery, and the thyroglobulin may not come down to normal levels after surgery. Finding a subcentimeter recurrent disease in the paratracheal area may not only be difficult, but hazardous in relation to injury to the parathyroid glands and recurrent laryngeal nerve. We must recognize that removal of this mini-volume recurrent disease does not guarantee complete cure, and the patient may return in the future with recurrent disease, either in the thyroid bed or in the ipsilateral or contralateral neck. We still need to adhere to the standard principle of medicine-first, do no harm-primum non nocere.

\section{References}

1. Bozec A, Dassonville O, Chamorey E, Poissonnet G, Sudaka A, Peyrottes I, Ettore F, Haudebourg J, Bussière F, Benisvy D, Marcy P-Y, Sadoul JL, Hofman P, Lassale S, Vallicioni J, Demard F, Santini J 2011 Clinical impact of cercival lymph node involvement and central neck dissection in patients with papillary thyroid carcinoma: a retrospective analysis of 368 cases. Eur Arch Otorhinolaryngol

2. Zeiger MA (2010) Evolution in the surgical management of welldifferentiated thyroid cancer or not: to dissect or not dissect the central lymph node compartment. J Surg Oncol 101(2):101-102

3. Cooper DS, Doherty GM, Haugen BR, Kloos RT, Lee SL, Mandel SJ, Mazzaferri EL, McIver B, Pacini F, Schlumberger M, Sherman SI, Steward DL, Tuttle RM (2009) Revised American Thyroid Association management guidelines for patients with thyroid nodules and differentiated thyroid cancer. American Thyroid Association (ATA) guidelines taskforce on thyroid nodules and differentiated thyroid cancer. Thyroid 19(11):1167-1214 [Erratum in: Thyroid 2010 Jun;20(6):674-675]

4. Shaha AR (2009) Prophylactic central compartment dissection in thyroid cancer: A new avenue of debate. Surgery 146(6): 1224-1227

5. Hughes DT, White ML, Miller BB et al (2010) Influence of prophylactic central neck dissection on postoperative thyroglobulin levels and radioiodine treatment in papillary thyroid cancer. Surgery 148(6):1100-1106 